https://doi.org/10.52340/lac.2021.674

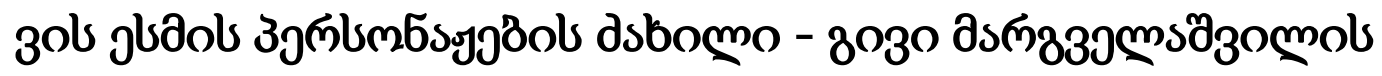

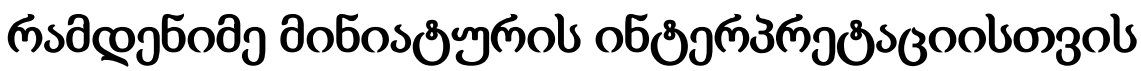

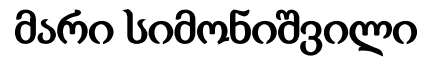

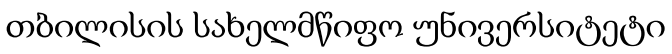 \\ e-mail: marisimonishvili13@gmail.com
}

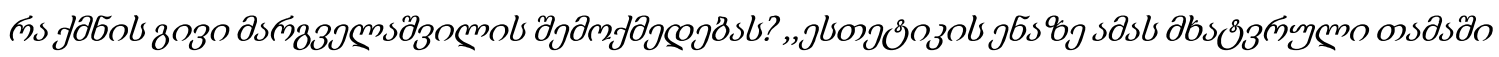

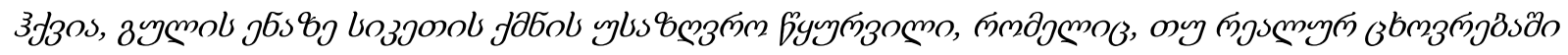

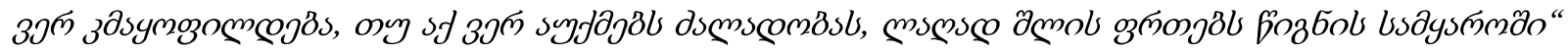

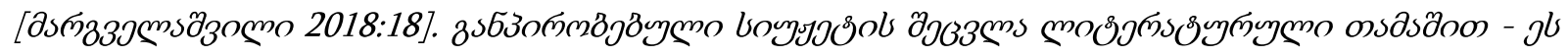

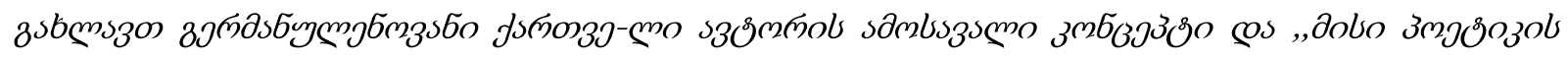

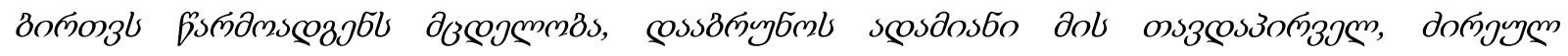

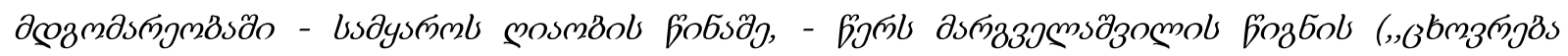

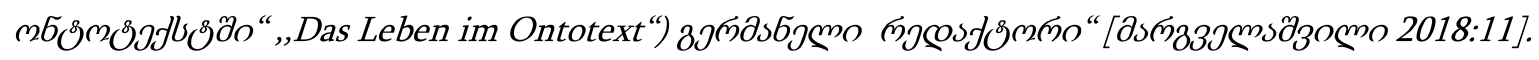

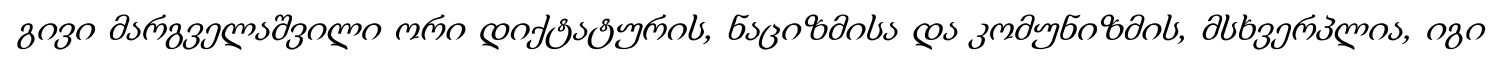

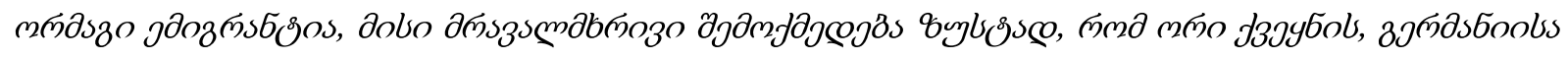

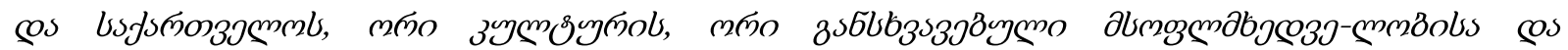

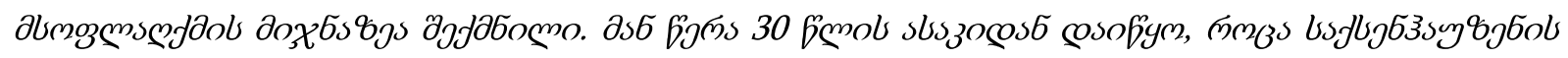

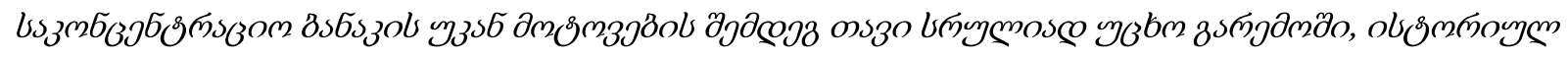

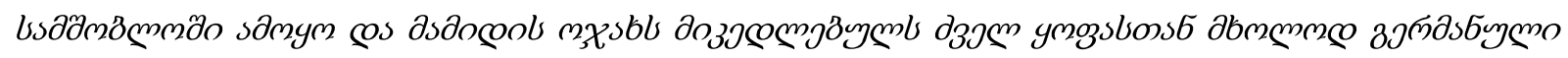

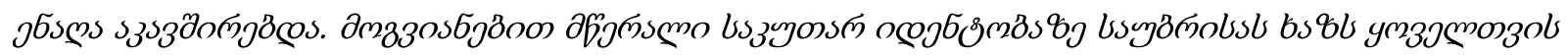

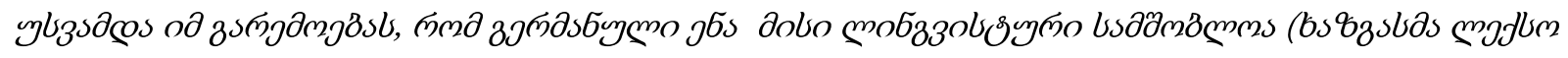

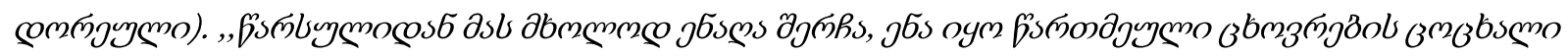

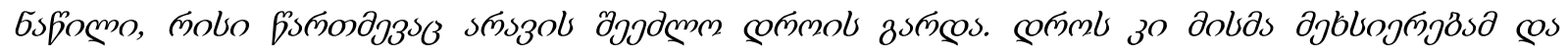




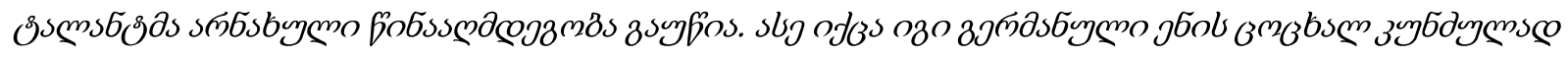

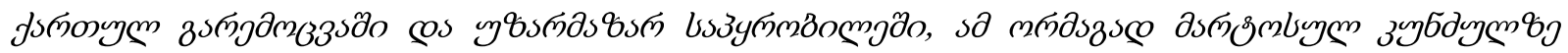

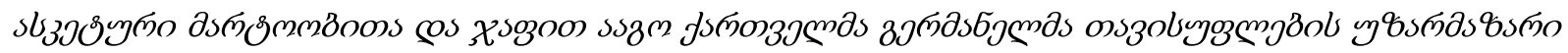

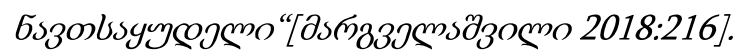

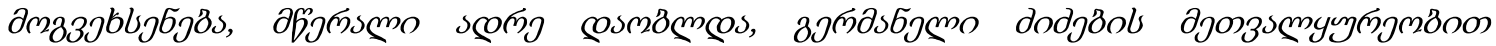

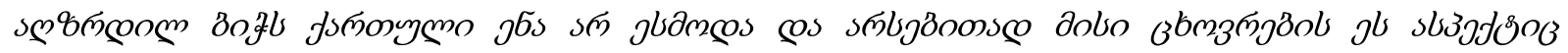

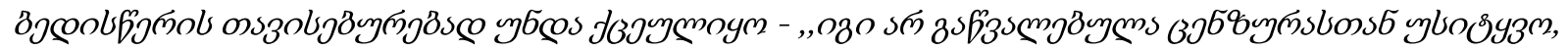

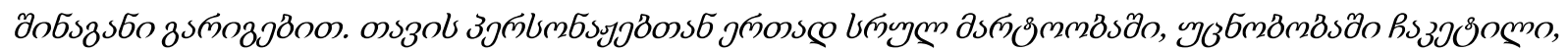

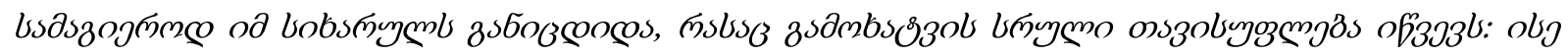

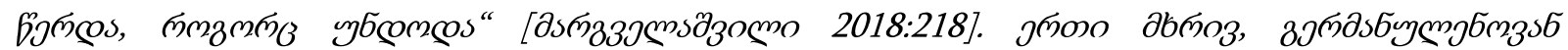

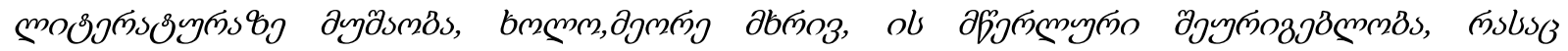

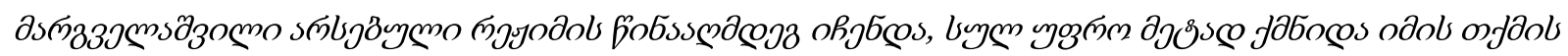

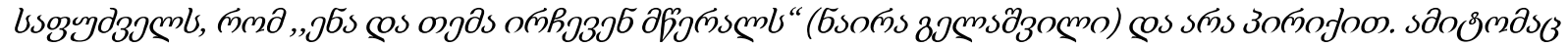

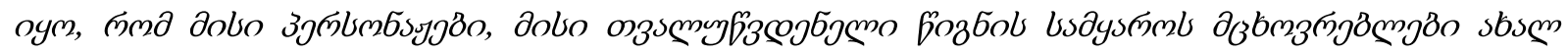

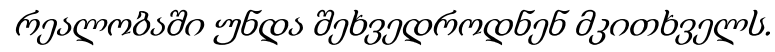

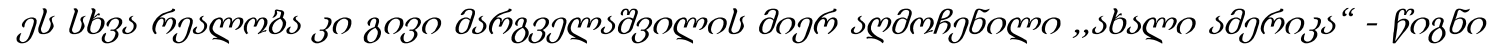

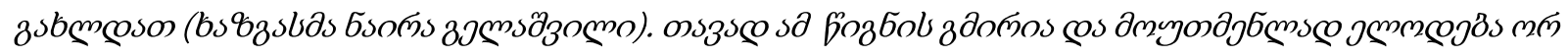

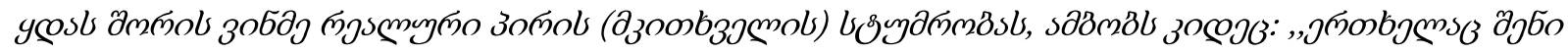

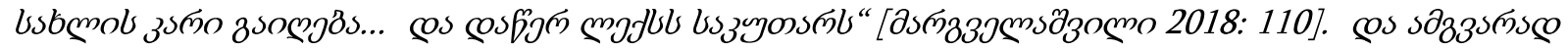

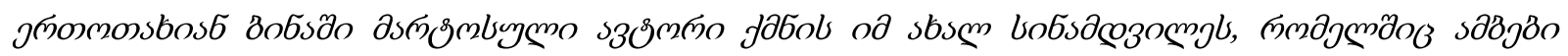

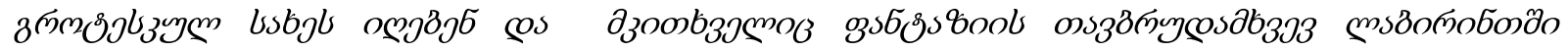
obsmsnong8s.

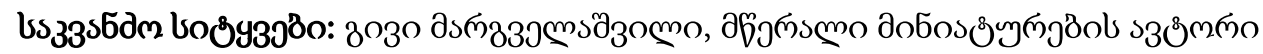

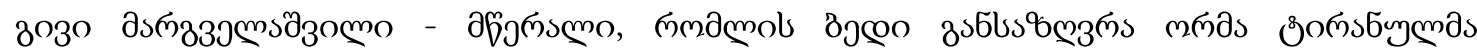

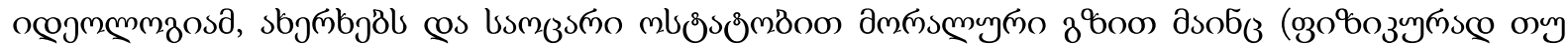

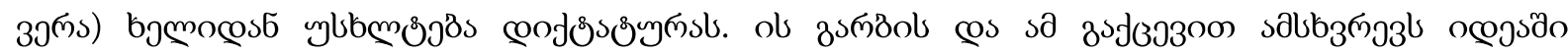

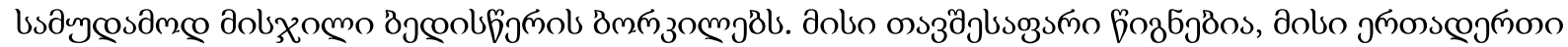




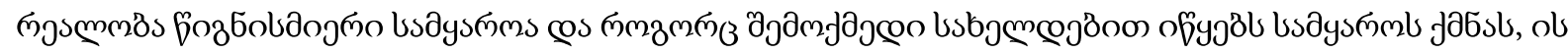

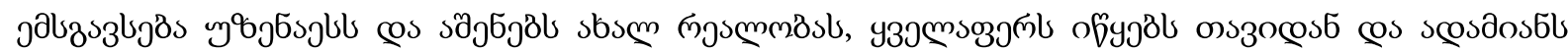

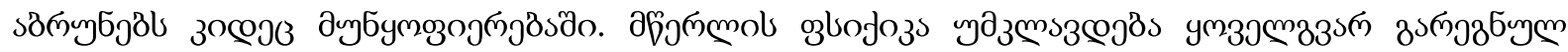

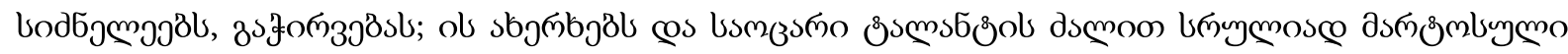

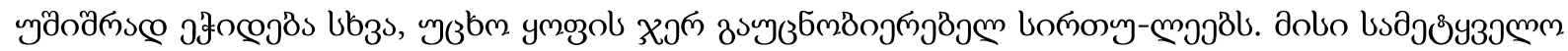

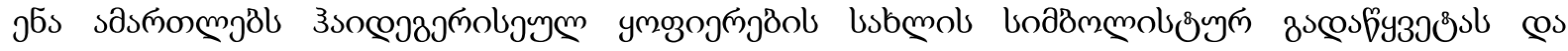

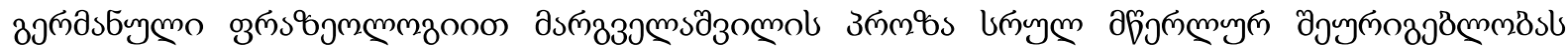

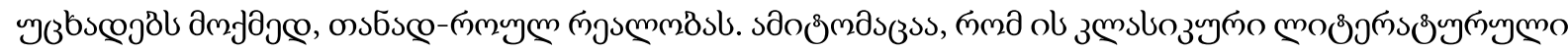

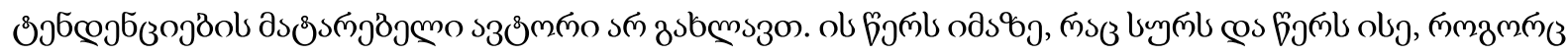

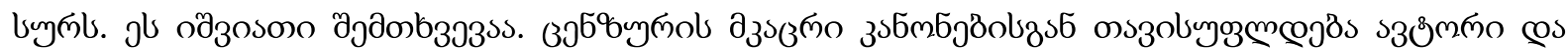

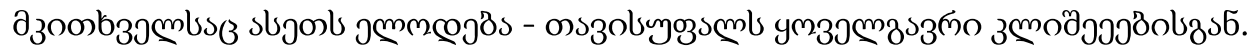

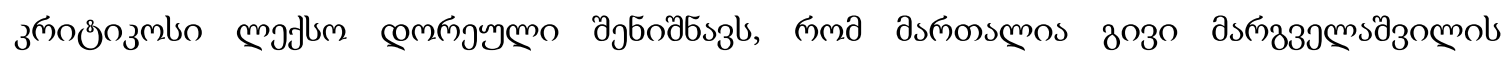

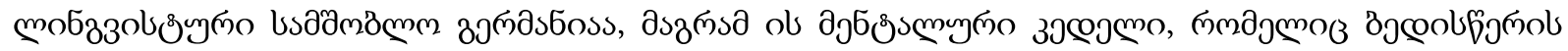

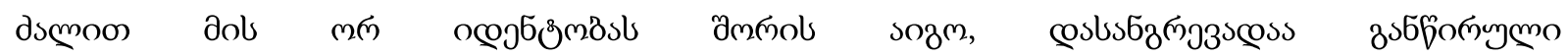
odos3oon39(http://arilimag.ge/\%E1\%83\%A5\%E1\%83\%94\%E1\%83\%97\%E1\%83\%98\%E1\%83\%9C\% E1\%83\%9D\%E1\%83\%91\%E1\%83\%90\%E1\%83\%96\%E1\%83\%90\%E1\%83\%AB\%E1\%83\%94\%E1\%8 3\%99\%E1\%83\%94\%E1\%83\%93\%E1\%83\%9A\%E1\%83\%98\%E1\%83\%A1\%E1\%83\%A0\%E1\%83\%94/ ?fbclid=IwAR1_mtun809Ez8p4X0RF9SDrmTRQZUf5zeI3FCCqJS0tI3Wi1XjksBbH5iQ).

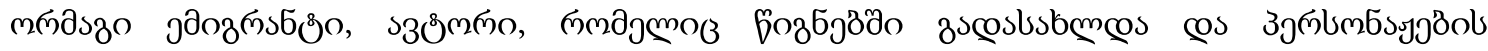

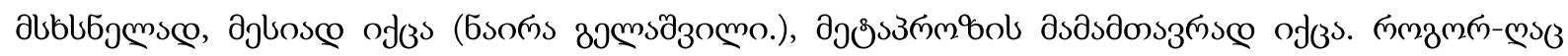

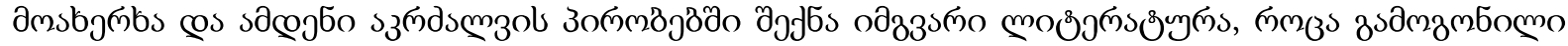

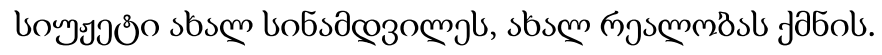

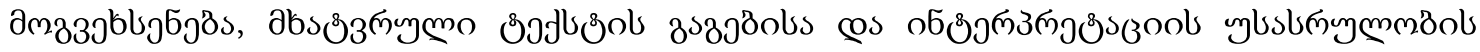

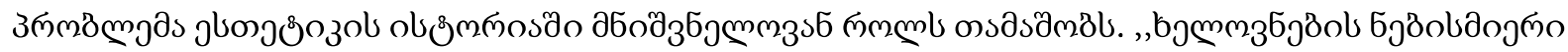

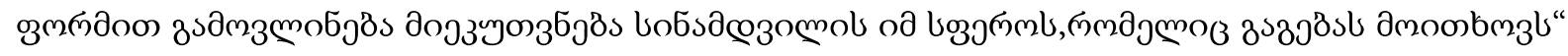

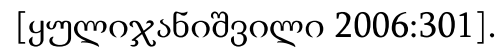

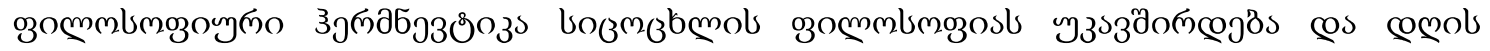

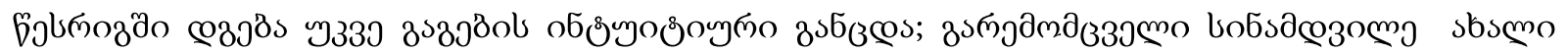

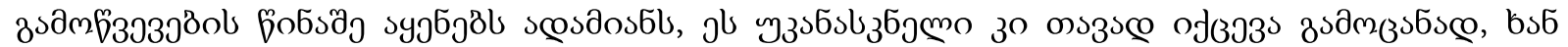




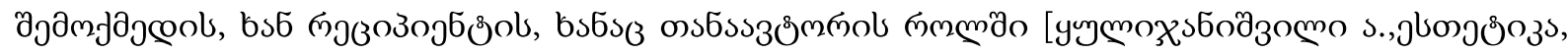

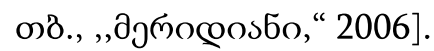

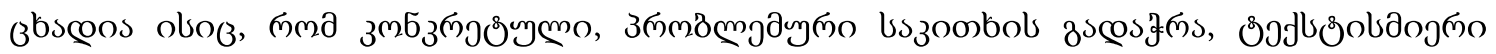

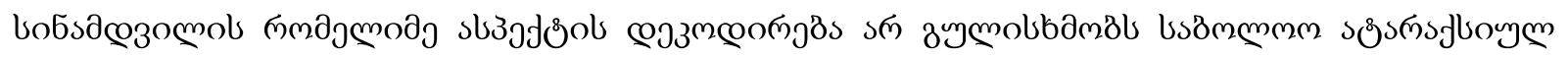

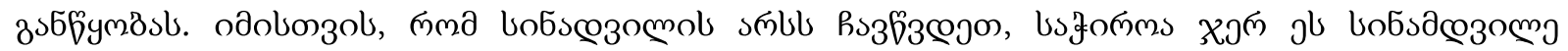

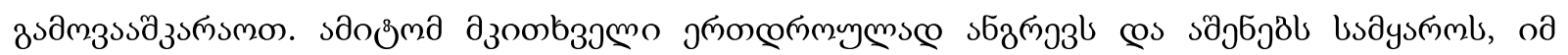

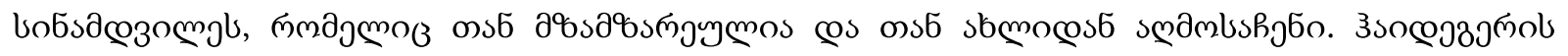

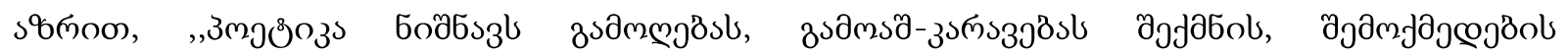

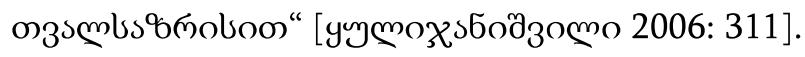

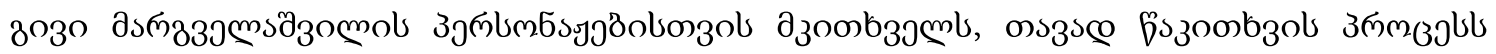

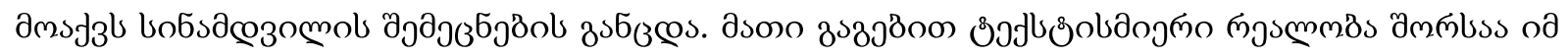

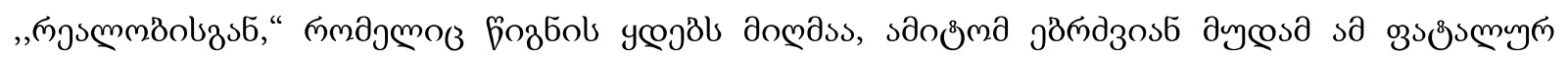

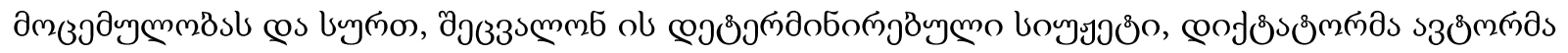

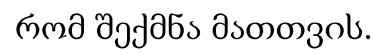

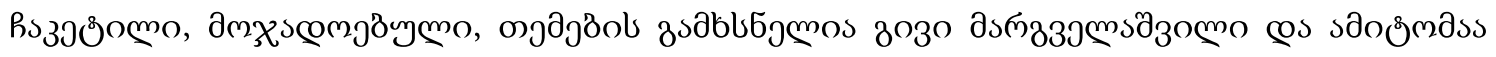

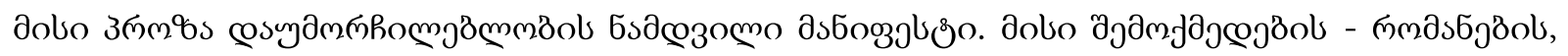

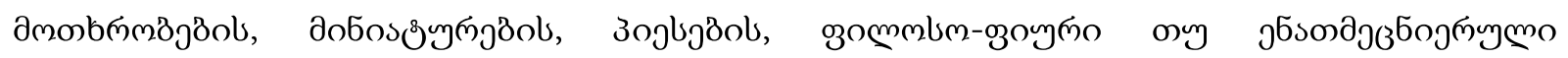

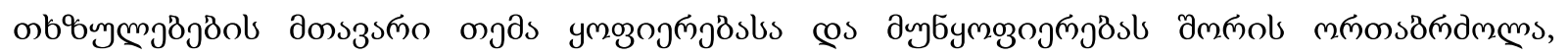

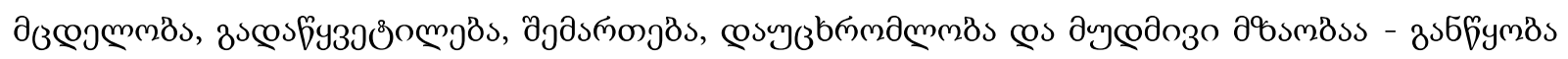

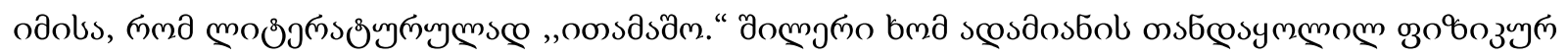

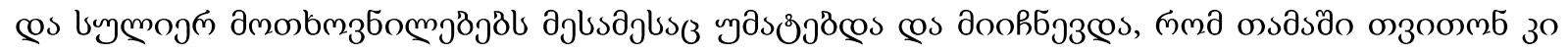

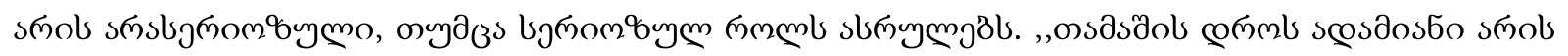

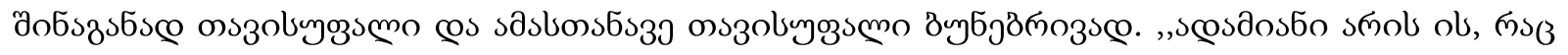

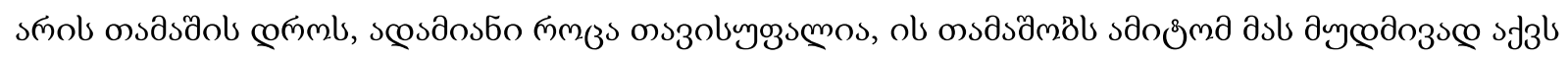

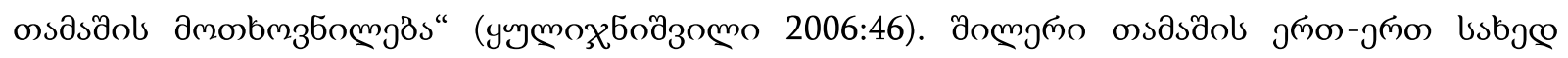

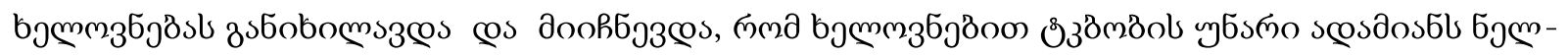

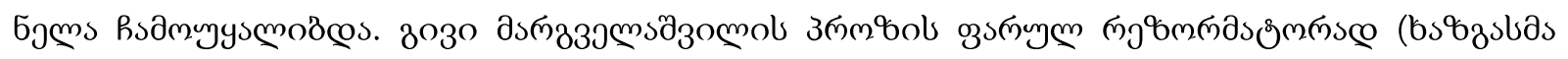

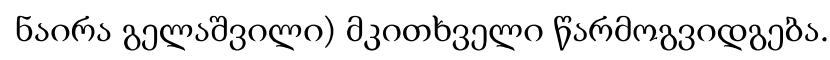




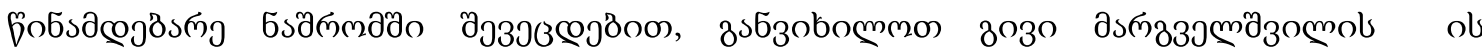

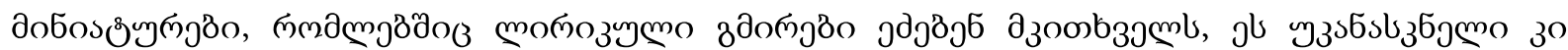

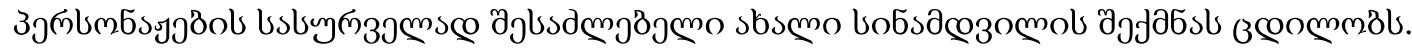

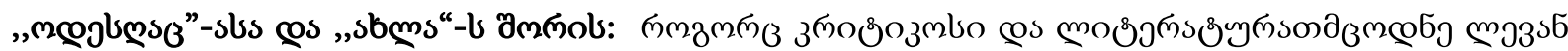

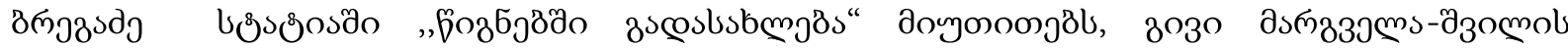

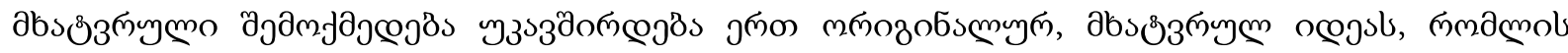

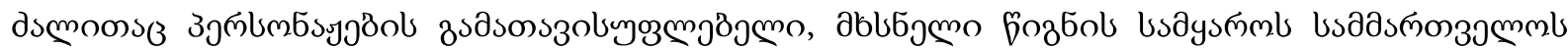

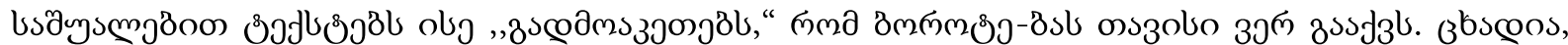

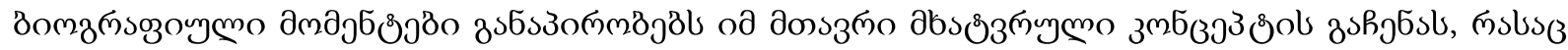

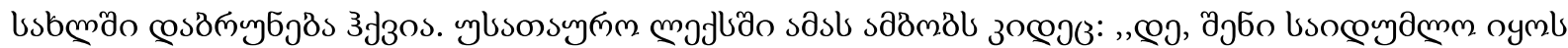

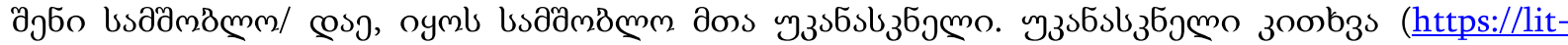
studies.blogspot.com

/2020/03/ blogpost13.html?fbclid=IwAR2yYCQrtXEOZJDYcBeYUWrcA9vRJ7GO8pf7rZGw02SyvboB4ycunz7 $\mathrm{jXsc})$.

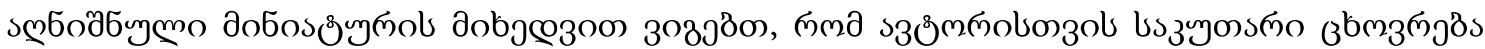

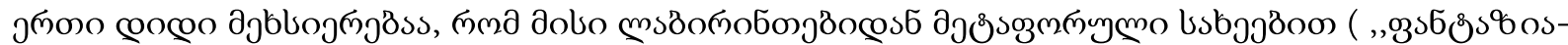

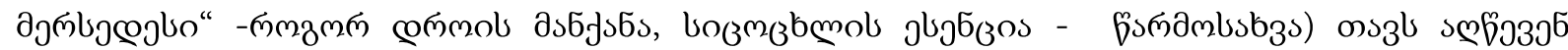

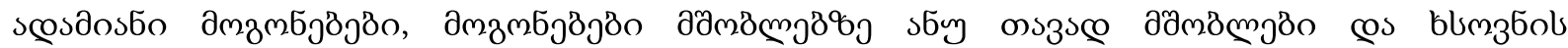

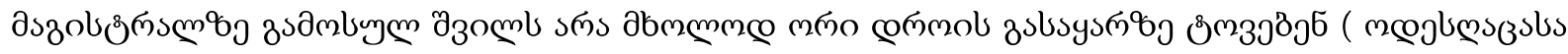

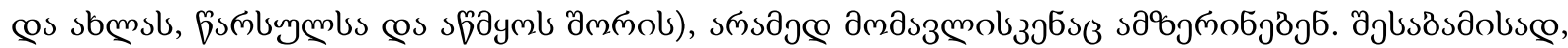

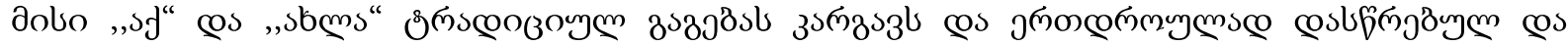

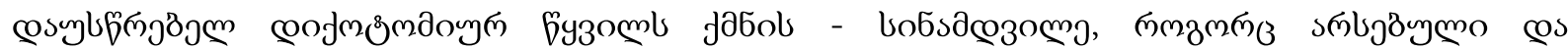

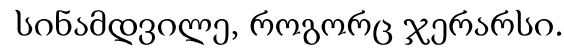

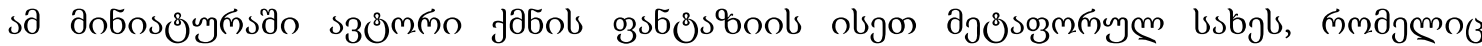

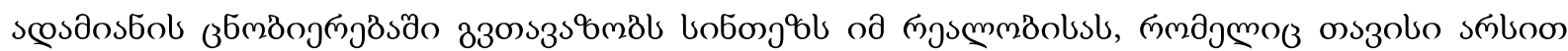

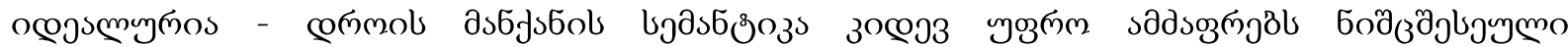

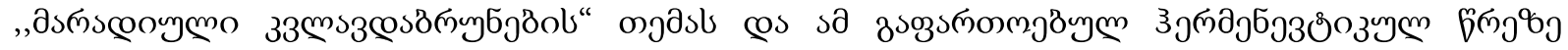

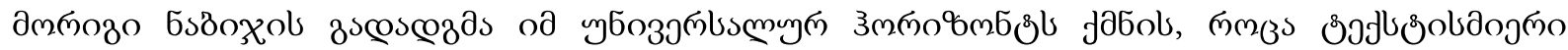




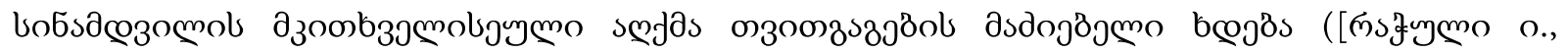

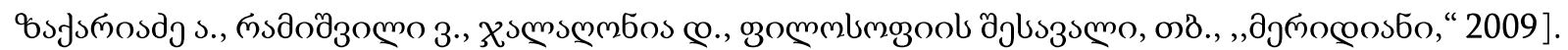

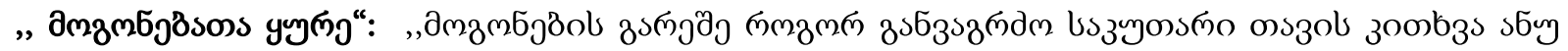

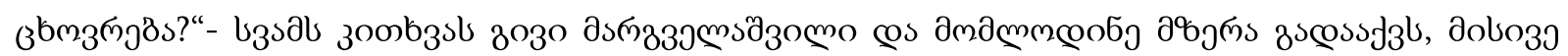

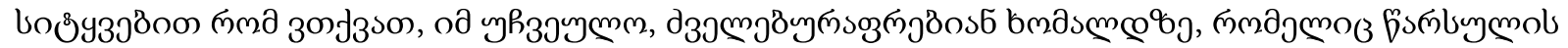

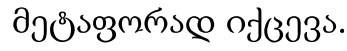

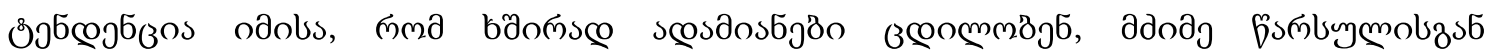

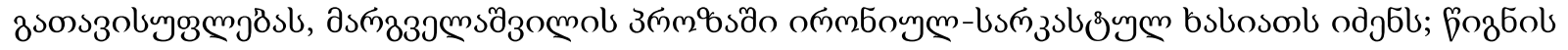

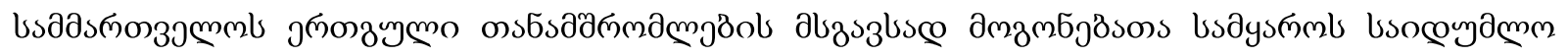

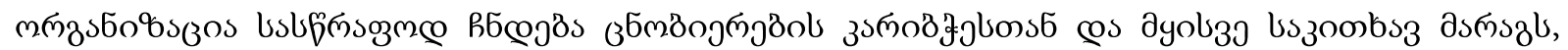

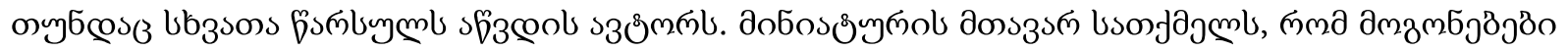

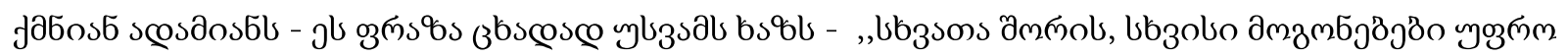

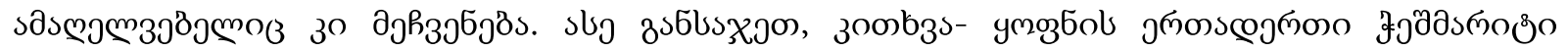

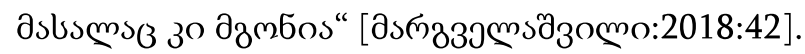

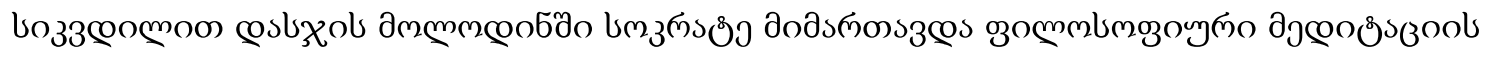

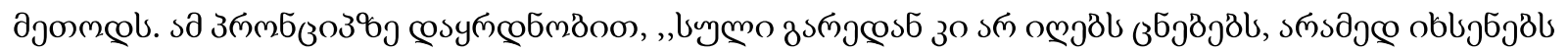

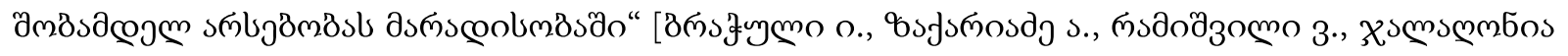

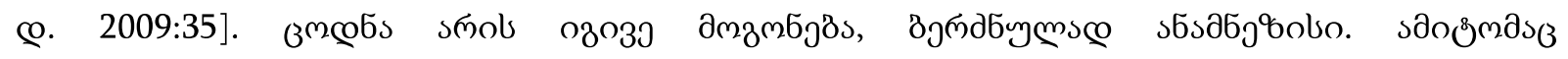

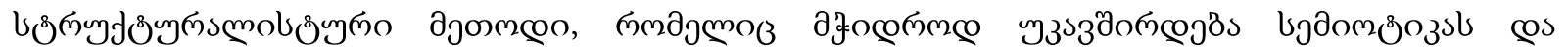

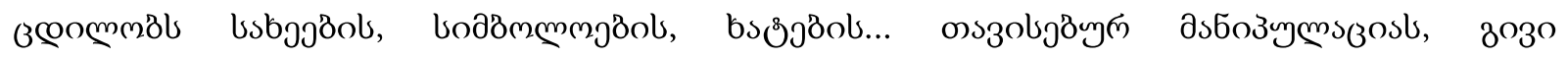

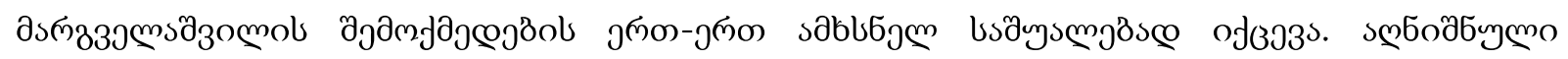

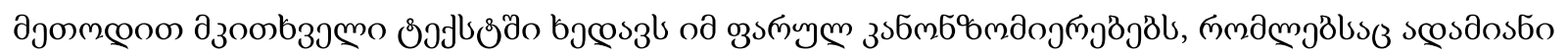

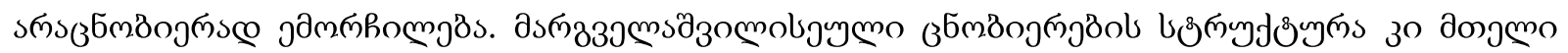

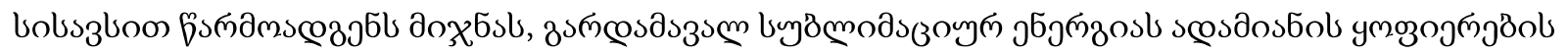

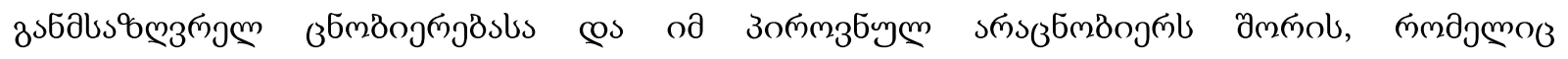

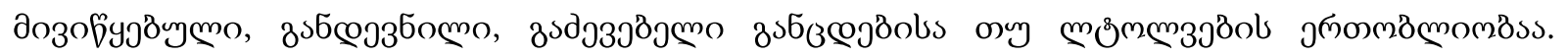

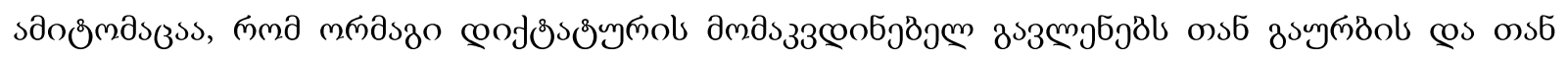

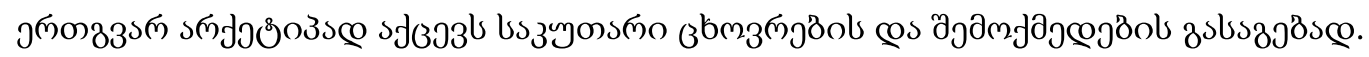




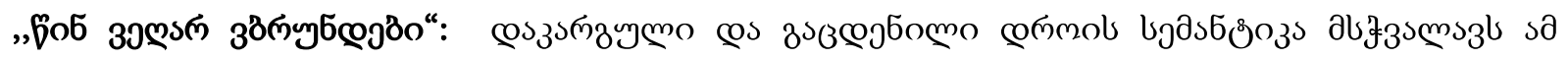

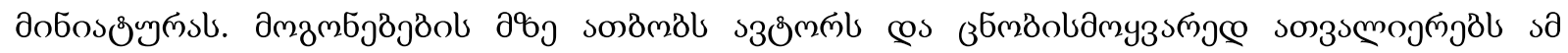

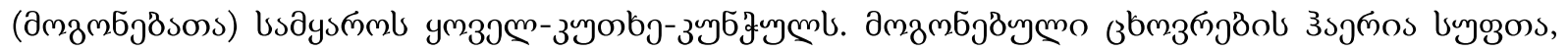

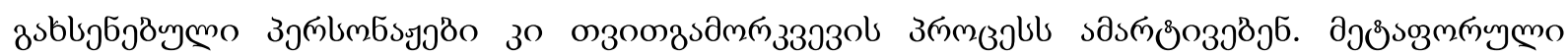

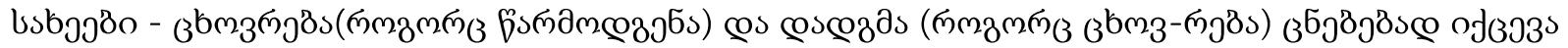

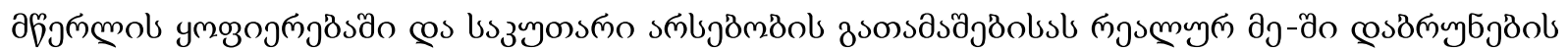

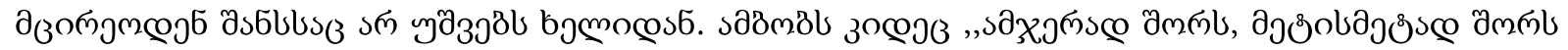

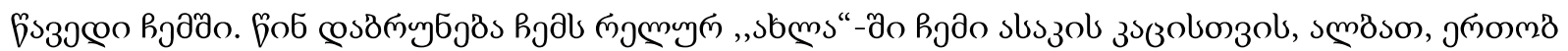

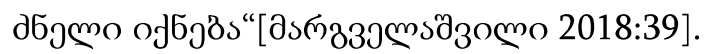

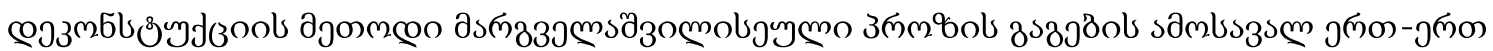

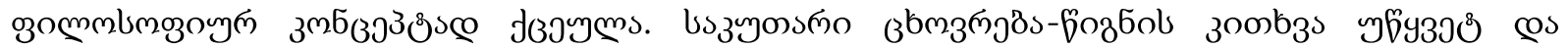

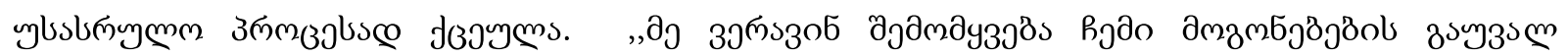

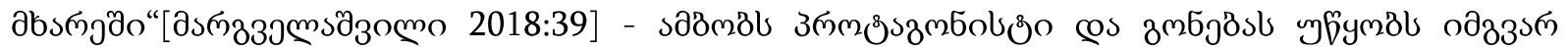

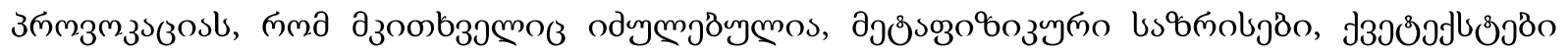

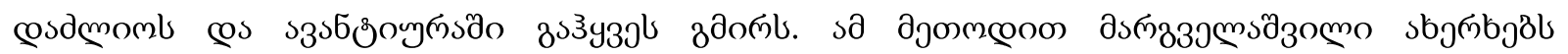

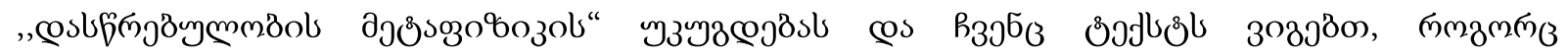

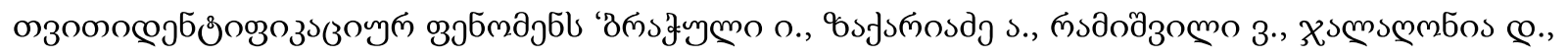

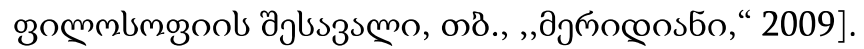

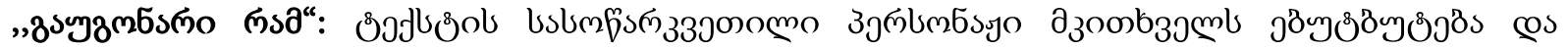

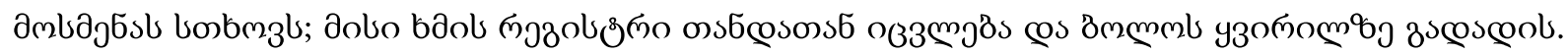

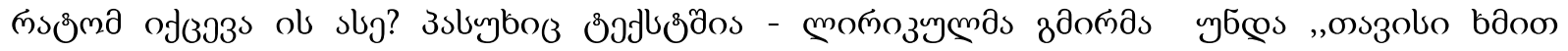

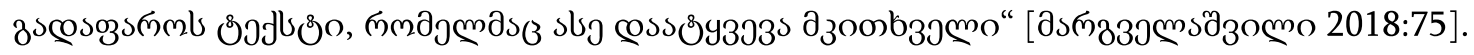

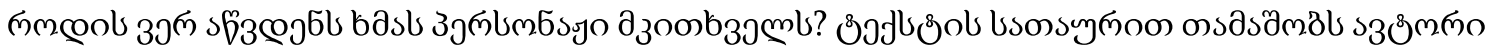

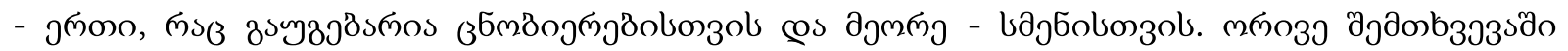

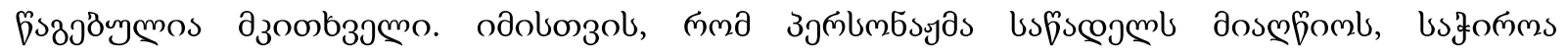

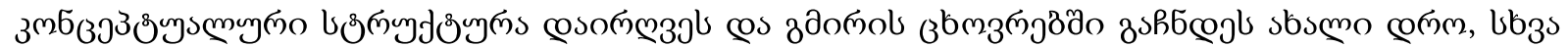

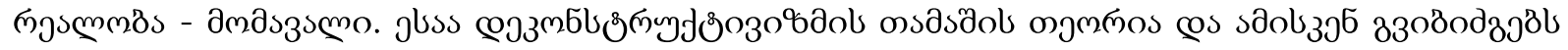

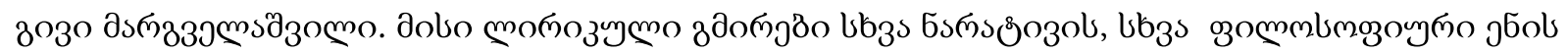

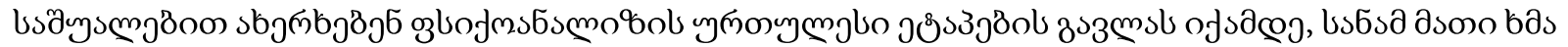




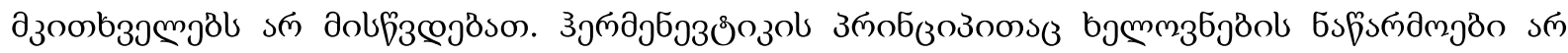

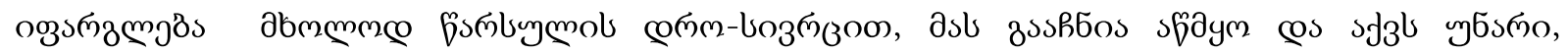

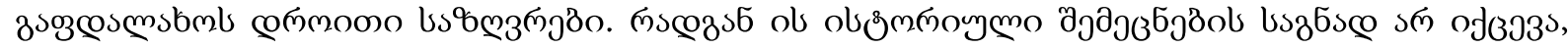

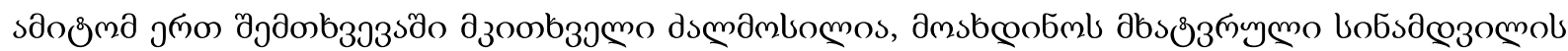

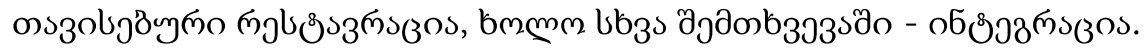

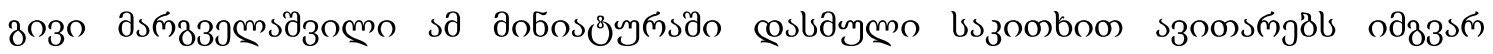

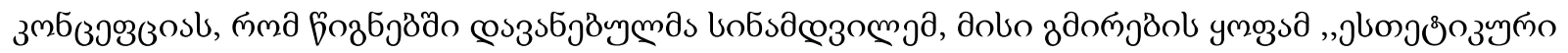

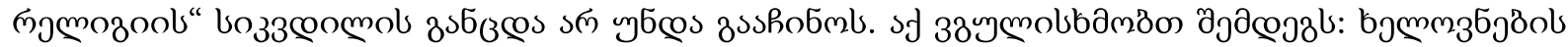

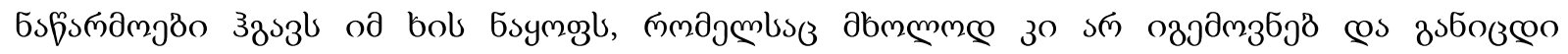

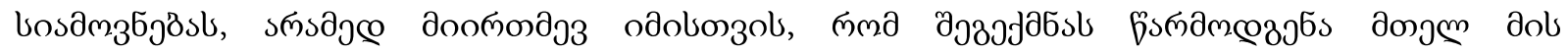

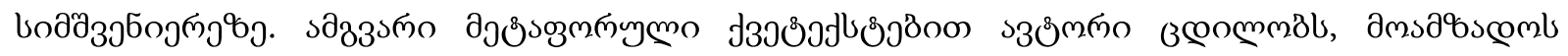

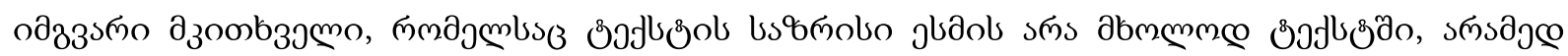

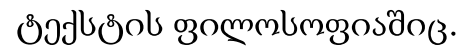

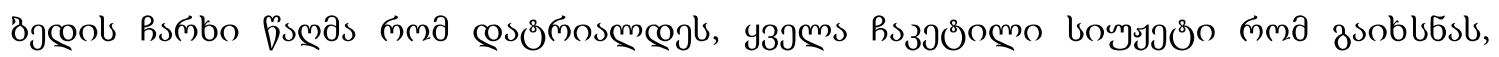

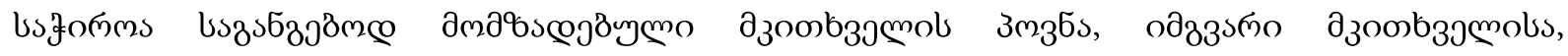

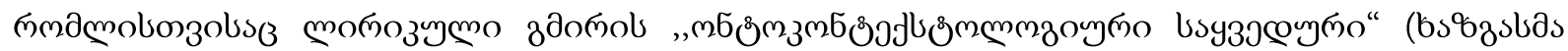

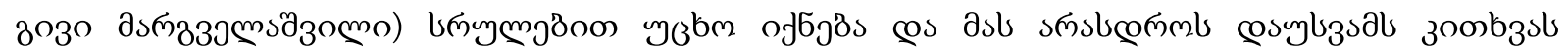

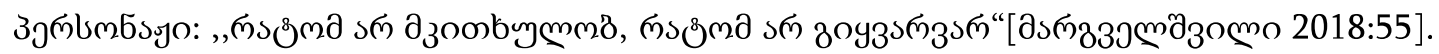

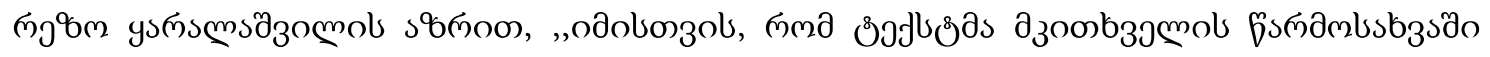

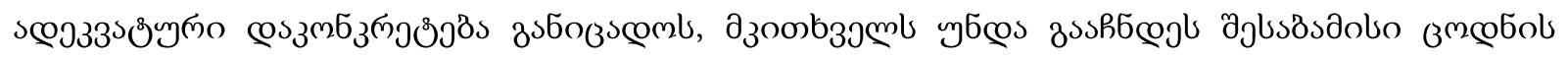

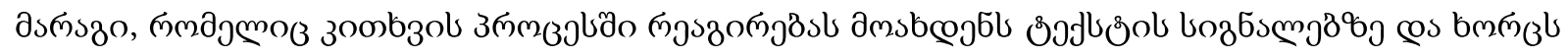

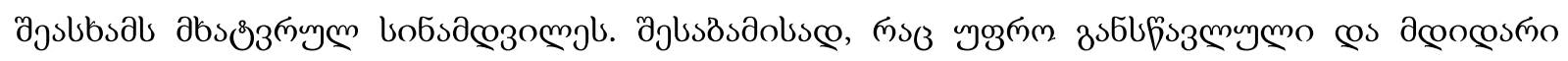

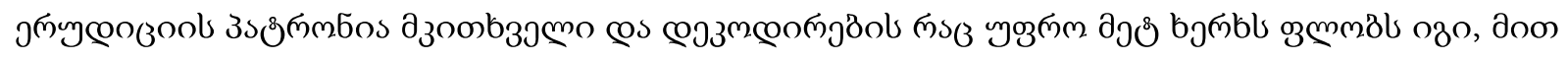

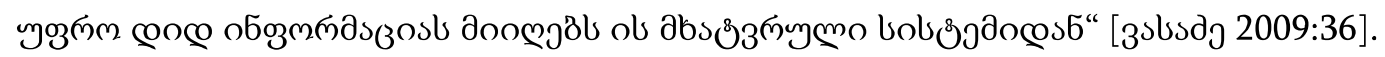

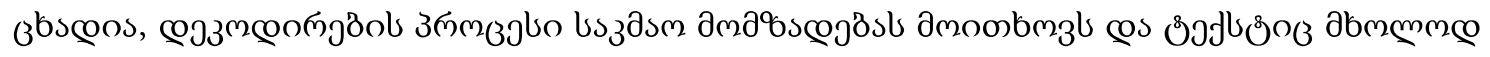

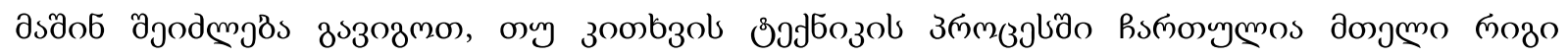

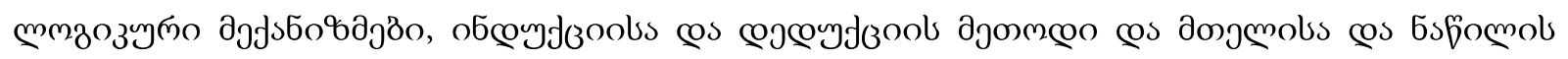

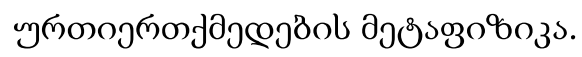




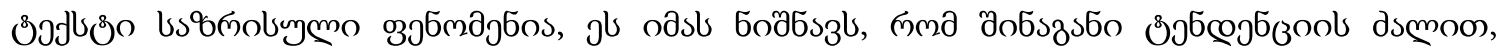

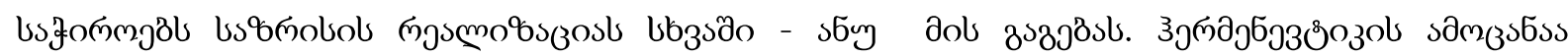

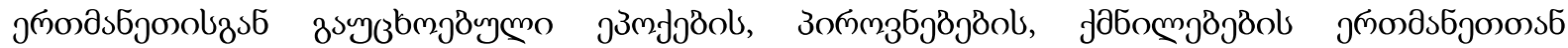

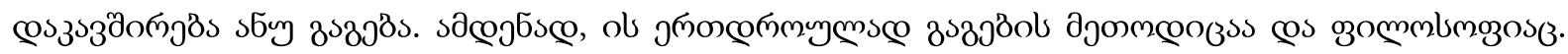

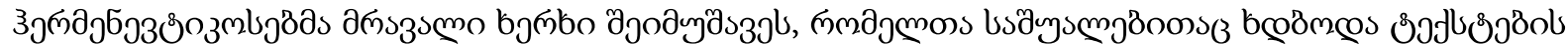

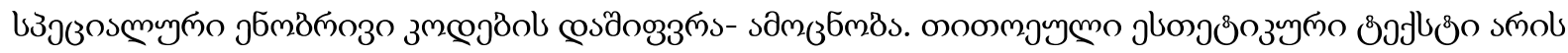

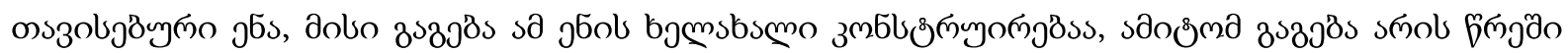

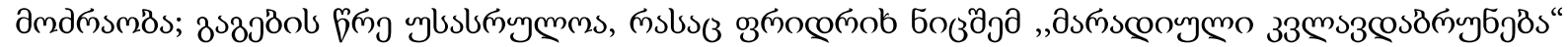

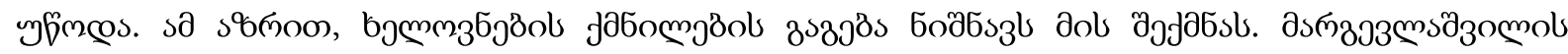

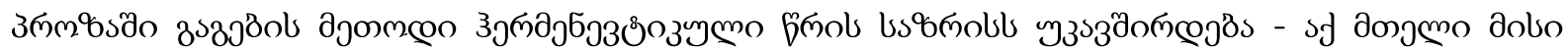

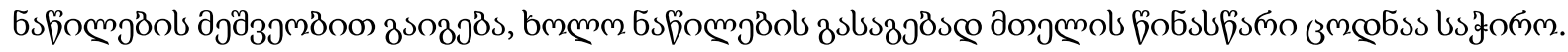

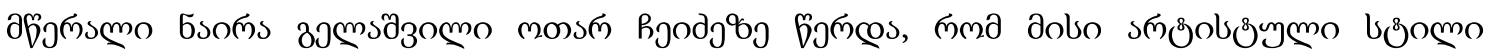

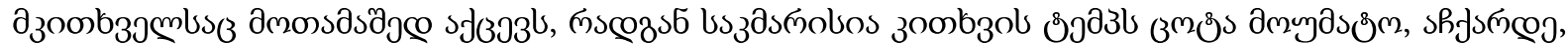

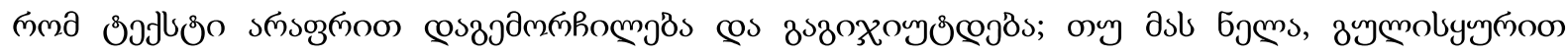

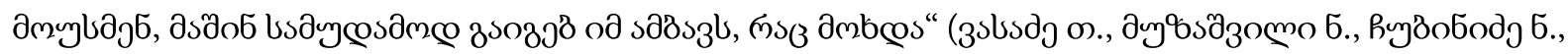

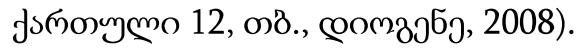

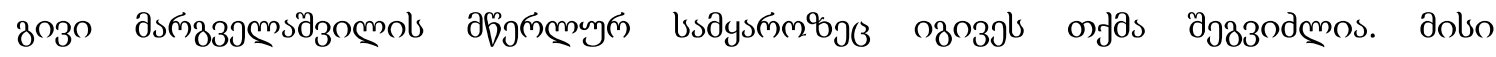

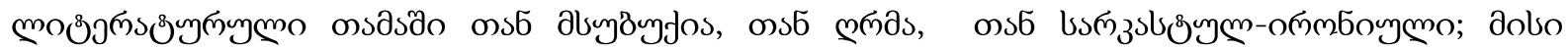

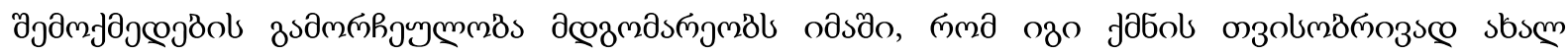

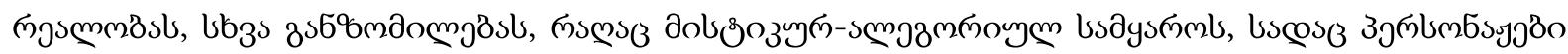

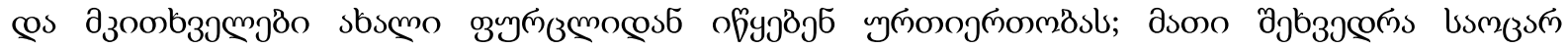

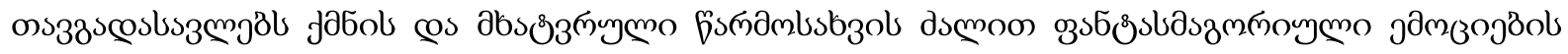

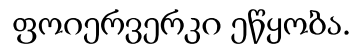

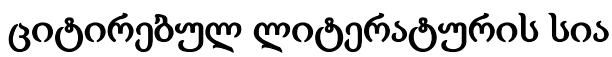

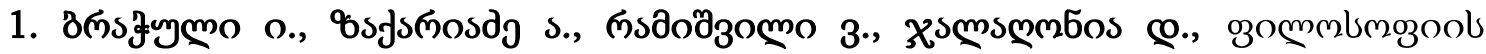

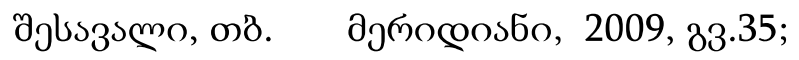

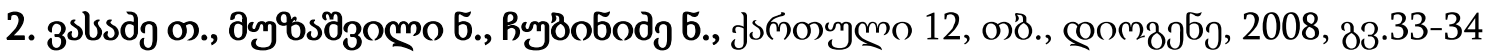




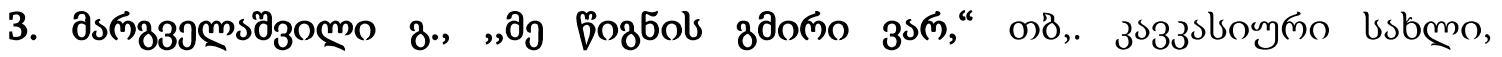
$2018,33.39,42,55,75$

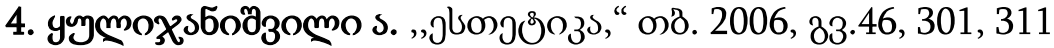

\section{öozmomzmsozos:}

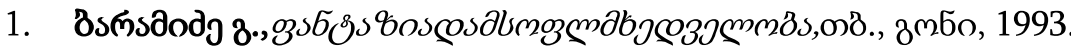

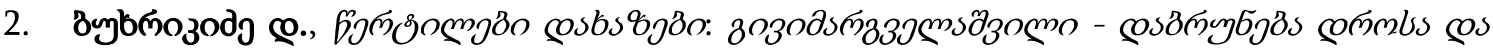

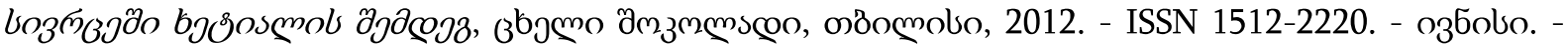
N80. - 33.118-119.

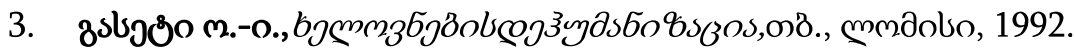

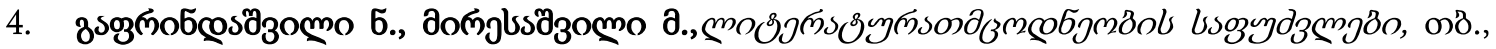

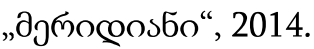

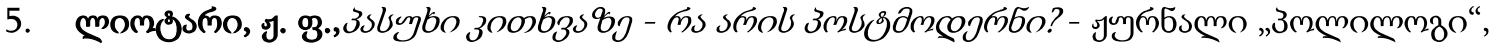
कరింmoluo, 1994, N4, 33. 195-207.

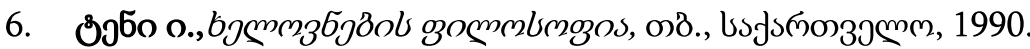

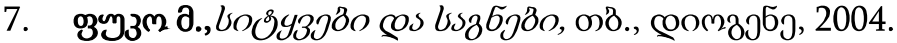

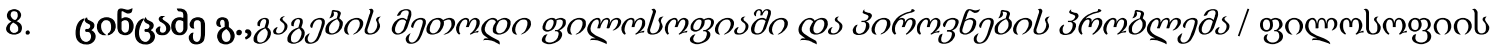

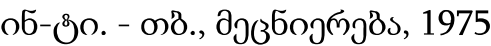

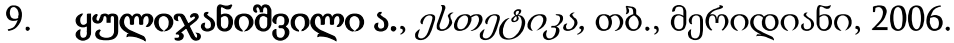

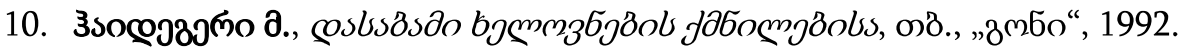

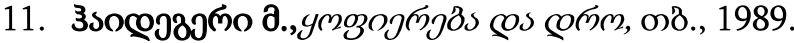

12. Делез Жиль, Фантазм и современная литература, Логика смысла, Москва, 1998, Стр. 366440 .

13. Делез Жиль, Гваттари Феликс, Анти-Эдип, Екатеринбург: У-Фактория, 2007.

14. Eco Umberto, Zeichen / Einführung in einen Begriff und seine Geschichte, übers. Aus Ital. Von Günter Memmert, Suhrkamp Verlag, Frankfurt am Main, 1977

15. Gadamer H.-G., Der Anfang der Philosophie, uebers. Aus Italienisch von Joachim Schulte, Stuttgart 1993 (= Reclam Universal-Bibliothek Nr. 9495) 
Mari Simonishvili

Iv.Javakhishvili Tbilisi State University

\title{
Who Understand to Cry of the Characters - for the Interpratation of Several Miniatures of Givi Margvelashvili
}

\begin{abstract}
What creates Givi Margvelashvili's work? "In the language of aesthetics, this is called an artistic game, in the language of the heart, it creates an boundless thirst for goodness, which, if it is not satisfied in real life, if it can not eliminate violence here, spreads its wings in the world of books" (Margvelashvili 2018: 18). Changing the conditioned story with a literary game - this is the starting concept of the Germanspeaking Georgian author and "at the core of his poetics is an attempt to return man to his original, fundamental state - the openness of the world," writes Margvelashvili's book "Life in Ontotext" Das Leben im ") German editor" (Margvelashvili 2018: 11).

Givi Margvelashvili is a victim of two dictatorships, Nazism and Communism. He started writing at the age of 30, when after leaving the Saxenhausen concentration camp, he found himself in a completely foreign environment, in his historical homeland, and his aunt's family was connected to his old life with only German. Later, when writing about his own identity, the writer always emphasized the fact that the German language is his linguistic homeland (emphasis add lexo doreuli). "From the past, only language was selected for him, language was a living part of a deprived life, which no one could take away except time. At times, however, his memory and talent met with unprecedented resistance. This is how it became a living island of the German language in the Georgian environment and in a huge prison, on this doubly lonely island the Georgian-German built a huge oil rig of freedom with ascetic loneliness and hard work "(Margvelashvili 2018: 216).

As we know, the writer was sick earlier, the boy brought up under the supervision of German nannies did not understand the Georgian language and essentially this aspect of his life should have become a feature of fate - "he was not bothered by a wordless, internal deal with censorship. Locked in complete solitude with his characters, unknown, he experienced the joy that comes with complete freedom of expression: he wrote as he wanted "(Margvelashvili 2018: 218). On the one hand, working on German-language literature, and on the other hand, the literary disagreement that Margvelashvili showed against the current regime, increasingly formed the basis for saying that "language and theme choose the
\end{abstract}


writer" (emphasis added Naira Gelashvili) and not vice versa. That was why his characters, the inhabitants of his inaccessible book world, had to meet the reader in a new reality.

This other reality was the book "New America" discovered by Givi Margvelashvili (emphasis added by Naira Gelashvili). He is the hero of this book and he is looking forward to the visit of a real person (reader) between the two covers, he even says: "Once the door of your house is opened ... and write a poem of your own" (Margvelashvili 2018: 110). And thus in a one-room apartment the lone author creates a new reality in which the stories take on a grotesque look and the reader is also entangled in a dizzying labyrinth of fantasy.

Key words: Givi Margvelashvili, Writer, Author of miniatures

๓эзว 